\title{
Understanding the Lunar System Architecture Design Space
}

\author{
Dr. Dale C. Arney ${ }^{1}$ and Dr. Alan W. Wilhite ${ }^{2}$ \\ Georgia Institute of Technology/National Institute of Aerospace, Hampton, VA 23666 \\ David M. Reeves ${ }^{3}$ \\ NASA Langley Research Center, Hampton, VA 23681
}

\begin{abstract}
Based on the flexible path strategy and the desire of the international community, the lunar surface remains a destination for future human exploration. This paper explores options within the lunar system architecture design space, identifying performance requirements placed on the propulsive system that performs Earth departure within that architecture based on existing and/or near-term capabilities. The lander crew module and ascent stage propellant mass fraction are primary drivers for feasibility in multiple lander configurations. As the aggregation location moves further out of the lunar gravity well, the lunar lander is required to perform larger burns, increasing the sensitivity to these two factors. Adding an orbit transfer stage to a two-stage lunar lander and using a large storable stage for braking with a one-stage lunar lander enable higher aggregation locations than Low Lunar Orbit. Finally, while using larger vehicles enables a larger feasible design space, there are still feasible scenarios that use three launches of smaller vehicles.
\end{abstract}

\section{Introduction}

TN October 2009, the Review of U.S. Human Spaceflight Committee recommended that NASA pursue a flexible 1 path strategy, where capabilities and technologies are developed as humans explore incrementally more challenging destinations [1]. Along that flexible path, the lunar surface is one destination that could help prepare for an eventual human mission to Mars. International partners are also interested in pursuing human missions to the lunar surface, as identified in the latest Global Exploration Roadmap released by the International Space Exploration Coordination Group (ISECG) [2].

This paper explores options within the lunar system architecture design space, identifying performance requirements placed on the propulsive system that performs Earth departure within that architecture based on existing and/or near-term capabilities. The analysis explores performance requirements for various system architecture options, including rendezvous and aggregation locations, launch manifests, and lunar lander configurations. An overview of the design space options considered in this paper is included in Table 1. The mission that is analyzed assumes that a facility exists in the lunar vicinity to support a reusable Lander Crew Module (LCM) and that sufficient infrastructure exists on the lunar surface to support the crew (down-and-out lander).

The four aggregation locations assumed are Low Lunar Orbit (LLO), two High Elliptical Lunar Orbits (HELOs), and Earth-Moon L2 (E-M L2). These aggregation locations will provide points near the Moon for the lunar lander and crew elements to rendezvous before and after the surface mission. Also, the system architectures analyzed in this paper utilize a reusable crew module, which will remain at the aggregation location between lunar missions. It is assumed that a facility is located at this point to maintain and supply the reusable crew module. The launch vehicles considered in this study are consistent with the planned heavy lift vehicles for the near future. The $105 \mathrm{t}$ and $70 \mathrm{t}$ Space Launch System (SLS) configurations are in development by NASA [3], while the $53 \mathrm{t}$ launch vehicle is consistent with the advertised Falcon Heavy launch vehicle by Space Exploration Technologies Corporation (SpaceX) [4]. These payload capabilities include a 5 percent adapter mass and a 5 percent margin. Finally, three lunar lander configurations are considered, which are described in more detail in Section III. A two-stage lander, similar to the Apollo Lunar Module (LM), has a separate stage for descent from and ascent to the aggregation location. An Orbit Transfer Vehicle (OTV) could be added to this lander to transfer from the aggregation location to

\footnotetext{
${ }^{1}$ Research Engineer II, 100 Exploration Way, AIAA Member.

${ }^{2}$ Samuel P. Langley Distinguished Professor, 100 Exploration Way, AIAA Associate Fellow.

${ }^{3}$ Aerospace Engineer, Space Mission Analysis Branch, MS 462, AIAA Member.
} 
LLO and back, leaving the lunar lander to perform a less demanding set of maneuvers. The third lander configuration uses a propulsive stage to perform a portion of the descent burn before staging above the surface. Then, a single stage lunar lander performs terminal descent and landing as well as ascent to the aggregation location.

Table 1: Lunar System Architecture Design Space Options

\begin{tabular}{|l|cccc|}
\hline \multicolumn{2}{|c|}{ Parameter } & LLO & \multicolumn{4}{c|}{ Alternatives } \\
\hline Aggregation Location & HELO 1 & $\begin{array}{c}\text { HELO 2 } \\
(100 \times 10,000 \mathrm{~km})\end{array}$ & Earth-Moon L2 \\
\hline $\begin{array}{l}\text { Launch Vehicle Payload } \\
\text { Capability }\end{array}$ & $105 \mathrm{t}$ & $70 \mathrm{t}$ & $53 \mathrm{t}$ \\
\hline Number of Launches & 2 & 3 & \\
\hline Lander Configuration & Two-Stage & $\begin{array}{c}\text { Two-Stage with } \\
\text { OTV }\end{array}$ & $\begin{array}{c}\text { One-Stage with } \\
\text { Braking Stage }\end{array}$ \\
\hline
\end{tabular}

\section{Background}

To analyze the performance of the various architecture trade options, the suite of systems available must first be understood. Also, current and near-term capabilities must be identified to ensure that the architectures that are being analyzed are both feasible and viable in the near future. The launch vehicles were described in Section I as 105 t, 70 $\mathrm{t}$, and $53 \mathrm{t}$ variants. Those values are the payload masses to LEO, including a 5 percent adapter mass and 5 percent margin. The in-space elements are as follows:

1. Orion Crew Module (CM)

2. Orion Service Module (SM)

3. Braking Stage (either Orion SM or a Large Storable Stage (LSS))

4. Orbit Transfer Vehicle (OTV)

5. Lunar Lander (two-stage or one-stage), divided into a crew module and propulsive module(s)

6. Cryogenic Propulsive Stage (CPS)

\section{A. Orion Multi-Purpose Crew Vehicle}

The Orion Multi-Purpose Crew Vehicle (MPCV) consists of two elements: the Crew Module (CM) and the Service Module (SM), as shown in Figure 1. The CM Module is the capsule that supports and protects the crew during four mission phases: launch, in-space, re-entry, and landing. In the lunar architecture, the CM houses the crew from Earth to the aggregation location. After the surface mission, the crew returns to the CM, where it remains during the return trip. The gross mass of the CM used in this study is $9,820 \mathrm{~kg}$ [5].

The service module provides the propulsion to return to Earth (via the Trans-Earth Injection (TEI) burn), as well as systems and consumables (water, air, etc.) needed to support the crew during the in-space mission. The propulsion system in the SM uses Nitrogen Tetroxide (NTO)/ Monomethylhydrazine (MMH) propellant. The engine in this study is assumed to be similar to the Shuttle Orbital Maneuvering Engine (OME), which has a specific impulse of 316 seconds [6]. The Orion SM has an inert mass of 4,429 $\mathrm{kg}$ and a propellant capacity of 7,907 kg, adding to a gross mass of $12,337 \mathrm{~kg}$. The total launch mass of the Orion MPCV is 22,157 kg [5].

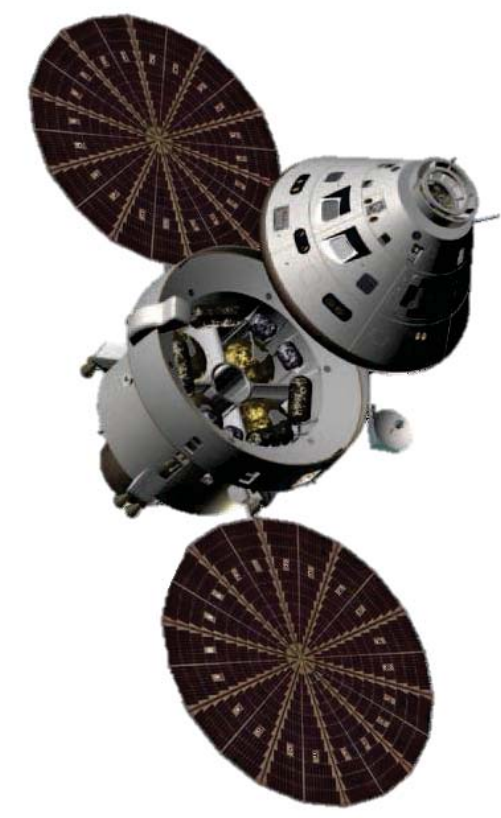

Figure 1: Artist's Rendering of the Orion MultiPurpose Crew Vehicle (Image Source: NASA) [5] 


\section{B. Braking Stage and Orbit Transfer Vehicle}

Two storable, in-space propulsive stages are included in the system architecture design space for this study. The first, a braking stage, performs a portion of the lunar descent burn from the aggregation location. The braking stage, as part of the "one-stage with braking stage" lander configuration, is also called a Lander Service Module (LSM). There are two options considered for the braking stage: an as-is Orion SM or a stretched version of the SM, called a Large Storable Stage (LSS). These two options are presented in Figure 2, where the LSS is simply an Orion SM with stretched propellant tanks. Figure 3 presents the curve of propellant mass fraction that is used as the propellant mass of the LSS increases. This curve is based off of typical behavior of a NTO/MMH propulsive stage as the propellant mass increases.

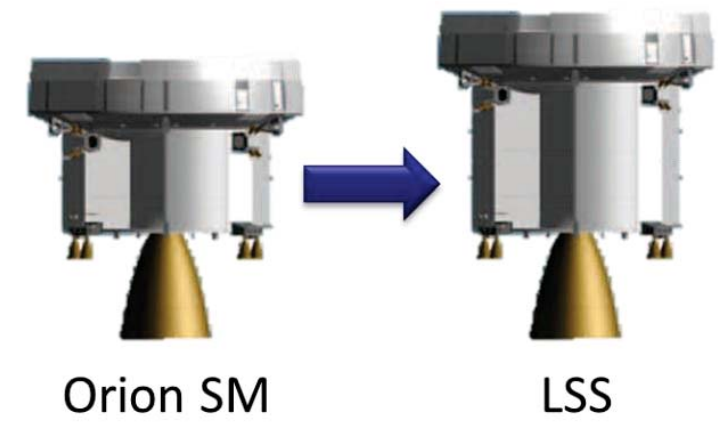

Figure 2: Large Storable Stage is a Stretched Orion Service Module

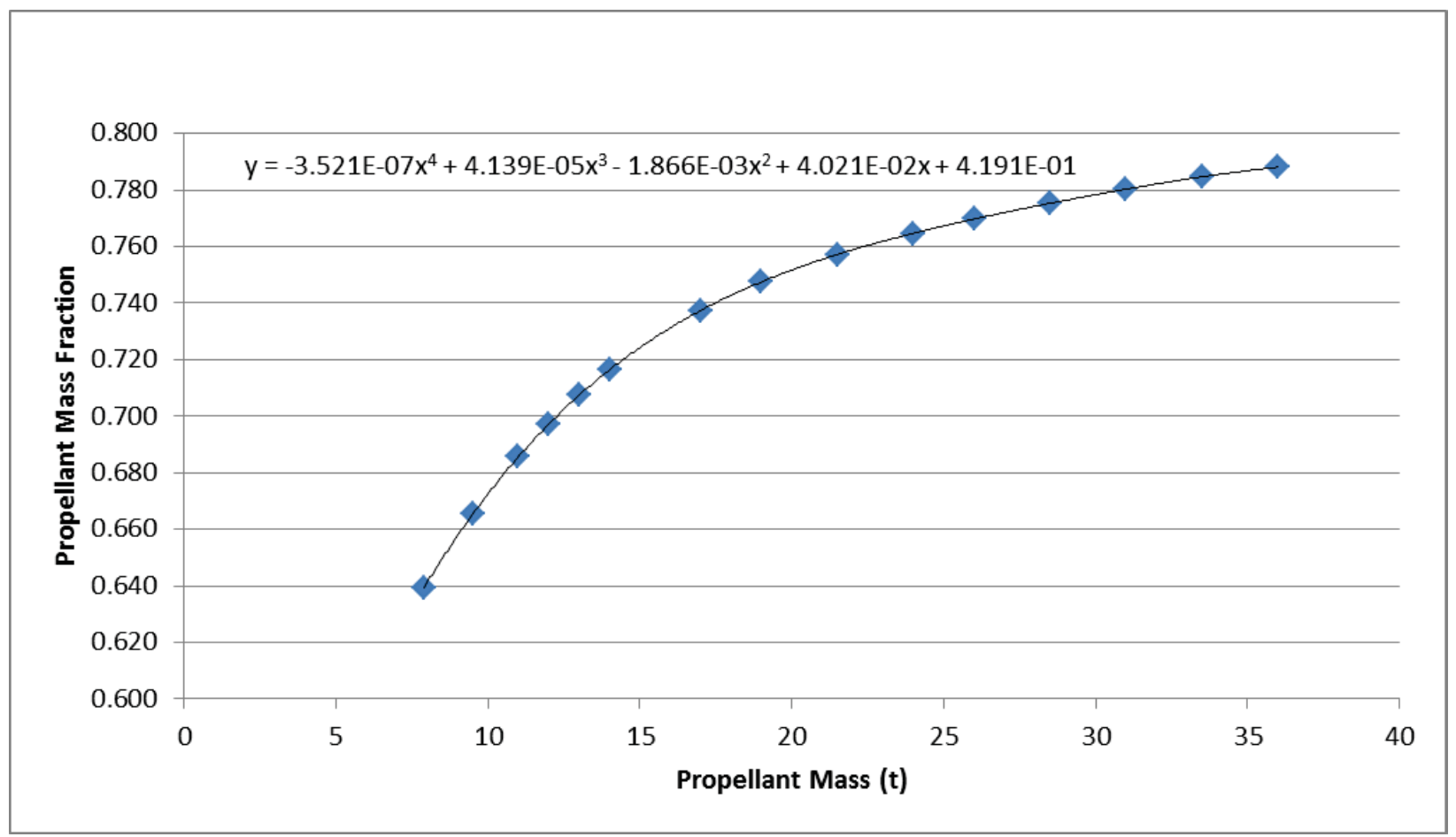

Figure 3: Propellant Mass Fraction of the LSS as Propellant Mass Varies

While the functionality of the braking stage is different than the Orion SM, the inert masses are not altered for this study. It is assumed by the authors that the additional subsystems needed to create a braking stage is similar in mass to the subtracted subsystems that are needed as a service module.

The Orbit Transfer Vehicle (OTV) transports the lunar lander from the aggregation location to LLO, where it loiters during the surface mission. After the surface mission, the crew rendezvous with the OTV and then returns to 
the aggregation location. This in-space stage must have storable propellant and be capable of multiple restarts. The same Orion SM and LSS stages can therefore be used as an OTV as well. Therefore, the same models mentioned above are used for the OTV.

\section{Lunar Lander}

Two types of lunar landers are considered in this study: two-stage and one-stage landers. Both types have a reusable Lander Crew Module (LCM) that remains at the aggregation location between lunar surface missions. This reusable LCM contains as much of the avionics, power, and other key subsystems as possible, thereby making the non-reusable portion of the lander as inexpensive as possible. As previously mentioned, it is assumed that a facility exists at that location that can support the LCM during those periods. In this analysis, the LCM mass is either assumed to be $3.0 \mathrm{t}$ or $5.0 \mathrm{t}$. This range is reasonable in the context of previous lander designs [7, 8, 9] and will show the impact of this module on the overall architecture. The remainder of the lander is called the Lander Propulsion Module (LPM). The LPM contains the tanks, structures, and propulsive systems required to access the lunar surface. The LPM can either be a two-stage system with a separate LPMD for Descent and LPMA for Ascent.

The lander Propellant Mass Fraction (PMF) is an independent variable in this study. Historical references are used to determine appropriate ranges for the two different configurations. For the two-stage lander, the Apollo lunar module and the Altair lunar lander were investigated. Table 2 presents the PMF for both the ascent and descent stages for each Apollo mission [7]. The PMF for the descent stage varies from 0.76 (when larger payloads were being delivered to the surface within the descent stage, e.g. the lunar rover) to 0.80. The variability of PMF for the ascent stage is smaller, with the PMF ranging from 0.52 to 0.53 . This inert mass in the Apollo PMF value includes the habitation, power, avionics, etc. that are included in the LCM in this analysis.

The Lunar Surface Access Module (LSAM) designed during the Exploration Systems Architecture Study (ESAS) was also a two stage lander [8]. The mass breakdown for the LSAM descent and ascent stages is presented in Table 3. The descent stage, which compared to the Apollo LM is a larger stage using Liquid Oxygen (LOX)/Liquid Hydrogen (LH2) propulsion, has a PMF of 0.73. The ascent stage, while similar in function to the Apollo LM, has a PMF of 0.45, which is much lower than the Apollo LM. This is perhaps due to conservatism in the modeling or differing requirements for the habitation (four crew instead of two). Recall that the lander crew module is split from the lander propulsive module in this study. Therefore, when sizing the LPM, the crew systems - including power, avionics, etc.-should not be included. The column titled "Modified Ascent Stage" presents the PMF of the LSAM ascent stage without the crew systems. The systems that remain are the propulsion systems, tanks, and structure. The PMF of this modified ascent stage is higher than that of the Apollo ascent stage (which still includes the crew systems) at 0.59 .

Table 2: Apollo Lunar Module Mass Breakdown [7]

\begin{tabular}{|c|c|c|c|c|c|c|c|}
\hline Mission: & 11 & 12 & 13 & 14 & 15 & 16 & 17 \\
\hline \multicolumn{8}{|l|}{ Descent Stage } \\
\hline Inert Mass (kg) & 4,483 & 4,875 & 4,650 & 4,712 & 6,179 & 6,083 & 6,155 \\
\hline Propellant Mass (kg) & 18,184 & 18,429 & 18,435 & 18,417 & 19,562 & 19,559 & 19,564 \\
\hline PMF & 0.8022 & 0.7908 & 0.7986 & 0.7963 & 0.7600 & 0.7628 & 0.7607 \\
\hline \multicolumn{8}{|l|}{ Ascent Stage } \\
\hline Inert Mass (kg) & 4,804 & 4,760 & -- & 4,691 & 4,690 & 4,704 & 4,729 \\
\hline Propellant Mass (kg) & 5,238 & 5,236 & -- & 5,225 & 5,237 & 5,243 & 5,262 \\
\hline PMF & 0.5216 & 0.5238 & -- & 0.5269 & 0.5276 & 0.5271 & 0.5267 \\
\hline
\end{tabular}


Table 3: Lunar Surface Access Module (LSAM) Mass Breakdown [8]

\begin{tabular}{|c|c|c|c|}
\hline Element: & $\begin{array}{l}\text { Descent } \\
\text { Stage }\end{array}$ & $\begin{array}{l}\text { Ascent } \\
\text { Stage }\end{array}$ & $\begin{array}{c}\text { Modified } \\
\text { Ascent Stage }\end{array}$ \\
\hline Inert Mass (kg) & 9,464 & 5,962 & 3,376 \\
\hline Propellant Mass (kg) & 25,591 & 4,847 & 4,847 \\
\hline PMF & 0.7300 & 0.4484 & 0.5894 \\
\hline
\end{tabular}

The one-stage with braking stage lander configuration uses an in-space propulsive stage (the LSM) to perform a portion of the descent burn. A single stage (the LPM) then performs the remainder of the descent and terminal landing. After the surface mission is complete, the LPM performs ascent to the aggregation location. This configuration is modeled after the Descent Assisted Split Habitat (DASH) lunar lander concept, presented in Figure 4 [9]. The DASH lander uses a LOX/LH2 Retro Module to perform up to 90 percent of the descent before it is discarded and crashes into the lunar surface. Then, the lander module performs terminal descent and landing. The payload module carries a habitat used for the surface duration, and the transport habitat supports the crew during descent and ascent. After the surface mission, the lander module performs ascent and the payload module is left on the surface. The architecture considered in this study assumes that the surface habitat is prepositioned to the lunar surface, and the only function of the lunar lander is to transport crew from the aggregation location to the lunar surface and back. However, the DASH transport habitat is functionally similar to the Lander Crew Module, and the DASH lander module is functionally similar to the one-stage Lander Propulsive Module.

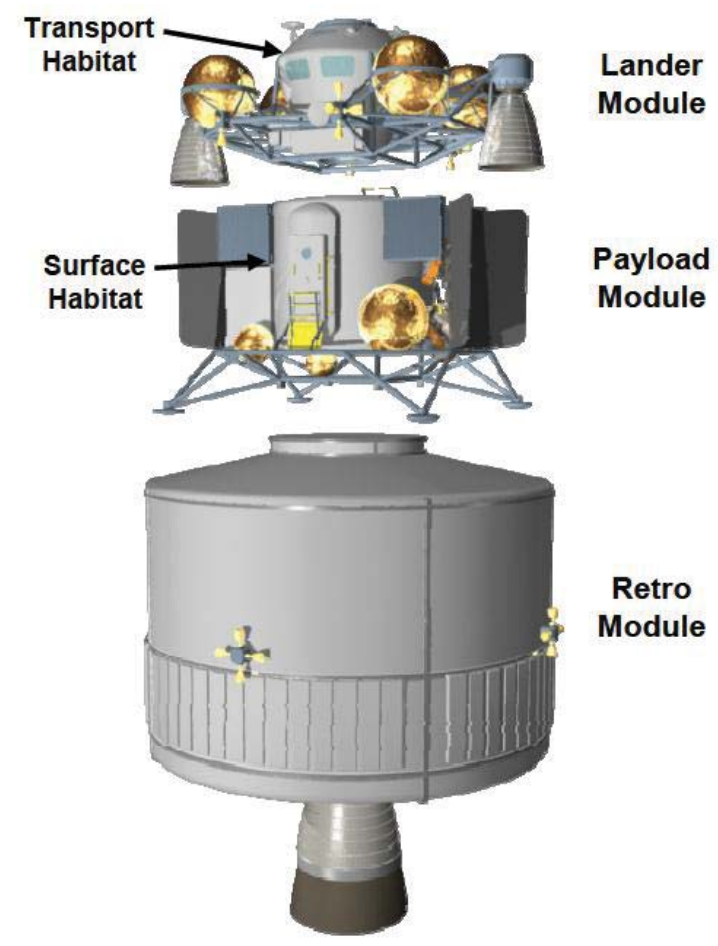

Figure 4: DASH Lunar Lander Concept [9]

The mass breakdown for the lander module of the DASH lander concept is presented in Table 4 [9]. Because this includes habitation elements in the masses, the "Modified Lander Module" removes those elements to only include the propulsive and structural elements that would apply to the LPM. Also, using photographic scaling relationships [10], the propellant demand for this element was increased to show the impact on PMF. For this range of propellant masses, the modified lander module (which is now analogous to the LPM) PMF varies between 0.77 and 0.81 . 
Table 4: DASH Lander Concept Mass Breakdown [9]

\begin{tabular}{|l|rrr|}
\hline \multicolumn{1}{|c}{ Element: } & $\begin{array}{l}\text { Lander } \\
\text { Module }\end{array}$ & $\begin{array}{l}\text { Modified } \\
\text { Lander } \\
\text { Module }\end{array}$ & $\begin{array}{l}\text { Larger } \\
\text { Lander } \\
\text { Module }\end{array}$ \\
\hline Inert Mass (kg) & 3,796 & 1,301 & 2,301 \\
\hline Propellant Mass (kg) & 4,389 & 4,389 & 9,697 \\
\hline PMF & 0.5362 & 0.7714 & 0.8082 \\
\hline
\end{tabular}

Table 5: Range of PMF for Various LPM Configurations

\begin{tabular}{|l|cc|}
\hline \multicolumn{1}{|c}{ Element Configuration } & Minimum PMF & Maximum PMF \\
\hline One-Stage LPM & 0.75 & 0.85 \\
\hline Two-Stage LPMD (descent) & 0.70 & 0.80 \\
\hline Two-Stage LPMA (ascent) & 0.55 & 0.65 \\
\hline
\end{tabular}

Because the LPM PMF is an independent variable in this analysis, having a reasonable range of PMF values for each system type is necessary. Based on the historical and conceptual lunar lander designs presented above, the ranges of PMF for each LPM configuration are presented in Table 5.

\section{Cryogenic Propulsive Stage}

The Cryogenic Propulsive Stage (CPS) is a LOX/LH2 propulsive stage that performs the Earth departure and lunar arrival burns. This stage must function during the transit to the aggregation location, which can vary from approximately five to ten days. The required PMF for the CPS to fit on the selected launch vehicle is calculated as a result of the analysis.

\section{Concepts of Operations}

The system architecture design space analyzed in this paper is divided into three concepts of operations based on the lunar lander configuration. The performance requirements are the same to maneuver between locations, but the systems used to traverse between those locations differ between the different concepts of operations. The same concept of operations is valid across multiple aggregation locations and two- or three-launch options for each lunar lander configuration.

Table 6 presents an estimate of the propulsive requirements to access the selected aggregation locations. Each row of the table defines the approximate $\Delta \mathrm{V}$ required to perform the given maneuver without any flight performance reserve. These values are estimates and are intended to be used to compare the rough locations and not analyze the performance requirements for a particular orbit. To select a particular orbit, more analysis must be done to incorporate abort modes, surface accessibility, etc.

Table 6: Estimated Propulsive Requirements for Selected Aggregation Locations

\begin{tabular}{|c|c|c|c|c|}
\hline Staging Orbit: & $\begin{array}{c}\text { LLO } \\
\text { (100 km circ.) }\end{array}$ & $\begin{array}{c}\text { HELO } \\
(100 \times 1,000 \mathrm{~km})\end{array}$ & $\begin{array}{c}\text { HELO } \\
(100 \times 10,000 \mathrm{~km})\end{array}$ & E-M L2 \\
\hline Trans-Lunar Injection (m/s) & 3,170 & 3,170 & 3,170 & 3,170 \\
\hline Lunar Orbit Insertion (m/s) & 880 & 730 & 370 & 250 \\
\hline E-M L2 to LLO Transfer (m/s) & -- & -- & -- & 720 \\
\hline Descent to Surface $(\mathrm{m} / \mathrm{s})$ & 2,000 & 2,200 & 2,600 & 2,000 \\
\hline Ascent from Surface (m/s) & 2,000 & 2,200 & 2,600 & 2,000 \\
\hline LLO to E-M L2 Transfer (m/s) & -- & -- & -- & 720 \\
\hline Trans-Earth Injection (m/s) & 900 & 750 & 400 & 280 \\
\hline Total & 8,950 & 9,050 & 9,140 & 9,140 \\
\hline
\end{tabular}




\section{A. Two-Stage Lunar Lander Configuration}

Figure 5 presents the concept of operations for the two-stage lunar lander configuration. First, a CPS (labeled CPS1) is delivered to Low Earth Orbit (LEO) with the LPMD and LPMA. The LCM is located at the aggregation point attached to the facility. In a three launch concept, propellant for the CPS1 can be delivered on a second flight and transferred over in LEO. CPS1 performs the Earth departure burn and the arrival burn to place the lunar lander into the aggregation location. The final launch delivers CPS2 and the crew in the Orion MPCV. CPS2 delivers the crew to the aggregation location, where the crew transfers to the lunar lander. The LPMD performs descent from the aggregation location. In the case of Earth-Moon L2, the descent stage also performs the transfer to LLO before descent. After the surface mission is complete, the LPMA performs ascent and transfers to the aggregation location. Once there, the crew transfers back to the Orion MPCV, where the Orion SM performs the Trans-Earth Injection (TEI) burn. The Orion CM then performs a direct entry at Earth.

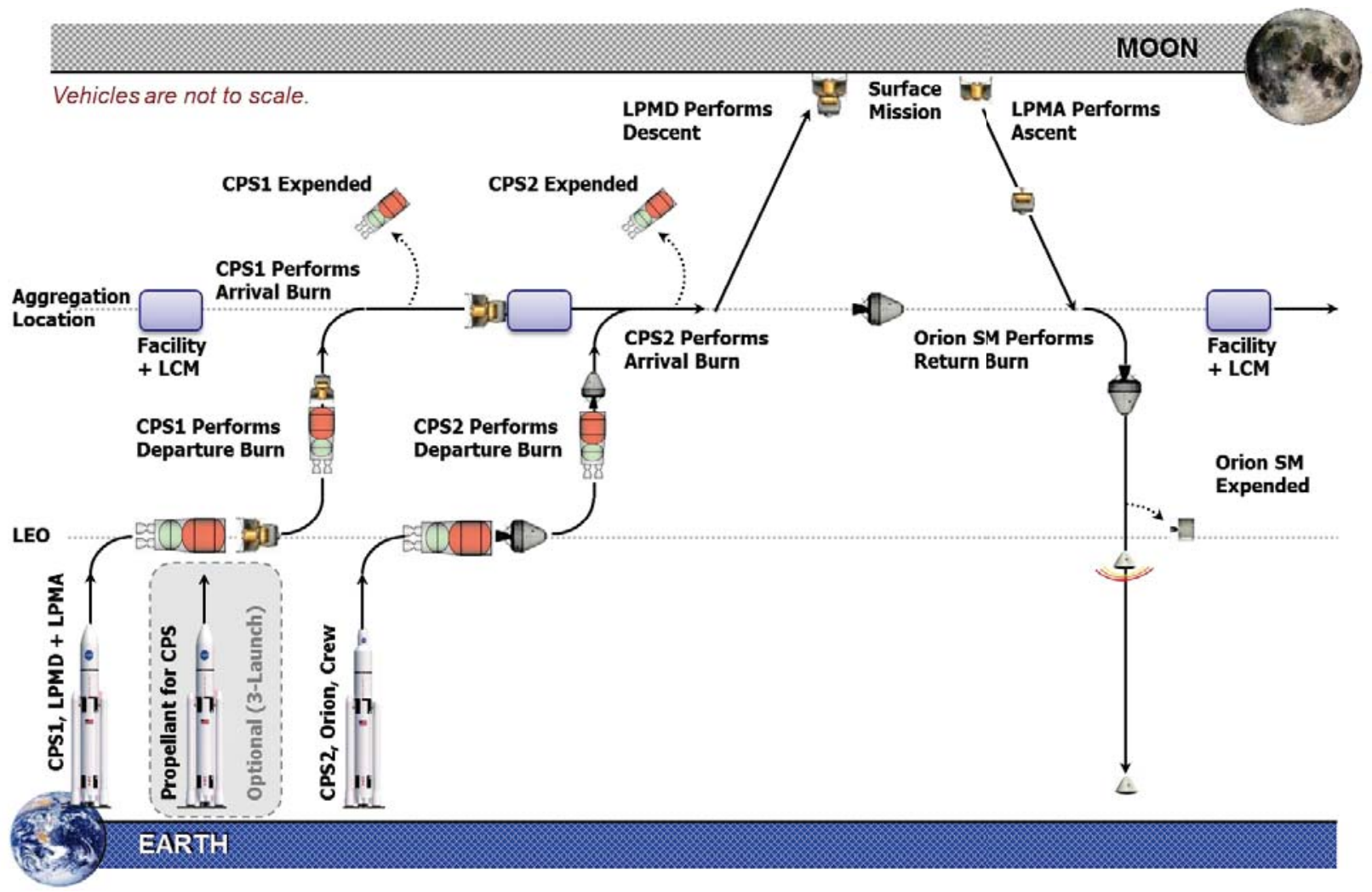

Figure 5: Two-Stage Lunar Lander Concept of Operations 


\section{B. Two-Stage Lander with Orbital Transfer Vehicle Configuration}

Figure 6 presents the concept of operations for the two-stage lunar lander with an Orbit Transfer Vehicle (OTV) configuration. The first launch delivers a partially filled CPS1 and the OTV to LEO. A second flight delivers propellant for the CPS1 along with the LPMD and LPMA. All of the two-stage lunar lander with an OTV scenarios utilize three launches. After it is filled with propellant, CPS1 performs the Earth departure burn and the arrival burn to place the lunar lander and the OTV into the aggregation location. The final launch delivers CPS2 and the crew in the Orion MPCV. CPS2 delivers the crew to the aggregation location, where the crew transfers to the lunar lander. The OTV transfers the lunar lander to Low Lunar Orbit (LLO), where it remains during the surface mission. The LPMD performs descent from LLO, and after the surface mission is complete, the LPMA performs ascent to the awaiting OTV. Once there, the OTV transfers the LPMA with the crew to the aggregation location. Then, the crew transfers back to the Orion MPCV, where the Orion SM performs the Trans-Earth Injection (TEI) burn. The Orion $\mathrm{CM}$ then performs a direct entry at Earth.

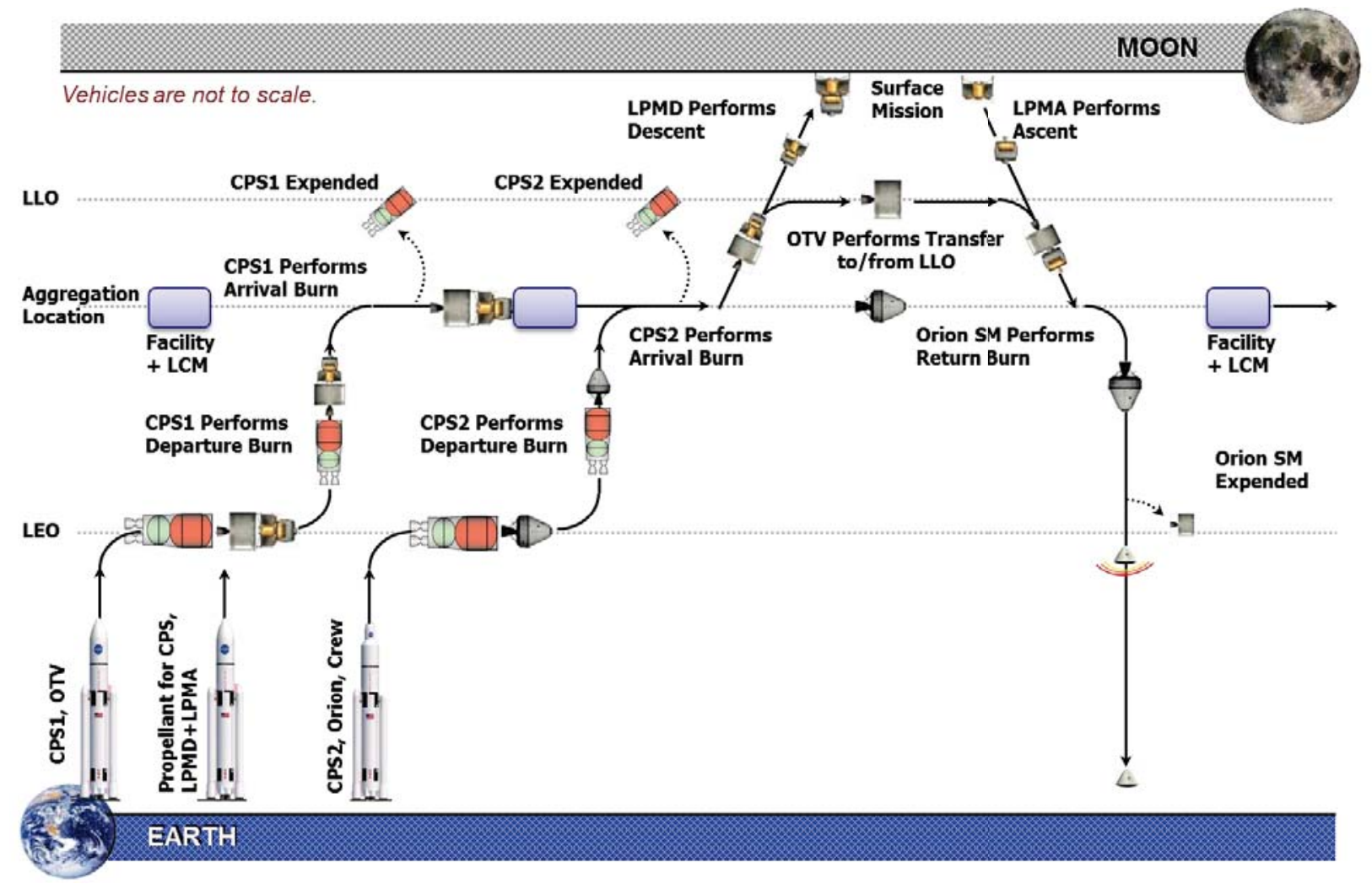

Figure 6: Two-Stage Lunar Lander with OTV Concept of Operations 


\section{One-Stage Lander with Braking Stage Configuration}

Figure 7 presents the concept of operations for the one-stage lunar lander with a braking stage configuration. CPS1 is delivered to LEO with the LPM and LSM (braking stage). In a three launch concept, propellant for the CPS1 can be delivered on a second flight and transferred over in LEO. CPS1 performs the Earth departure burn and the arrival burn to place the lunar lander into the aggregation location. The final launch delivers CPS2 and the crew in the Orion MPCV. CPS2 delivers the crew to the aggregation location, where the crew transfers to the lunar lander. The LSM performs a portion of the descent from the aggregation location. In the scenario where the Orion $\mathrm{SM}$ is used as the LSM, the amount of $\Delta \mathrm{V}$ is dictated by the fixed propellant load. In the scenario where an LSS is used as the LSM, the amount of $\triangle \mathrm{V}$ varies to minimize the total LSM and LPM mass. The LSM is staged above the lunar surface, and the LPM continues with terminal descent and landing. After the surface mission is complete, the LPM performs ascent and transfers to the aggregation location. Once there, the crew transfers back to the Orion MPCV, where the Orion SM performs the Trans-Earth Injection (TEI) burn. The Orion CM then performs a direct entry at Earth.

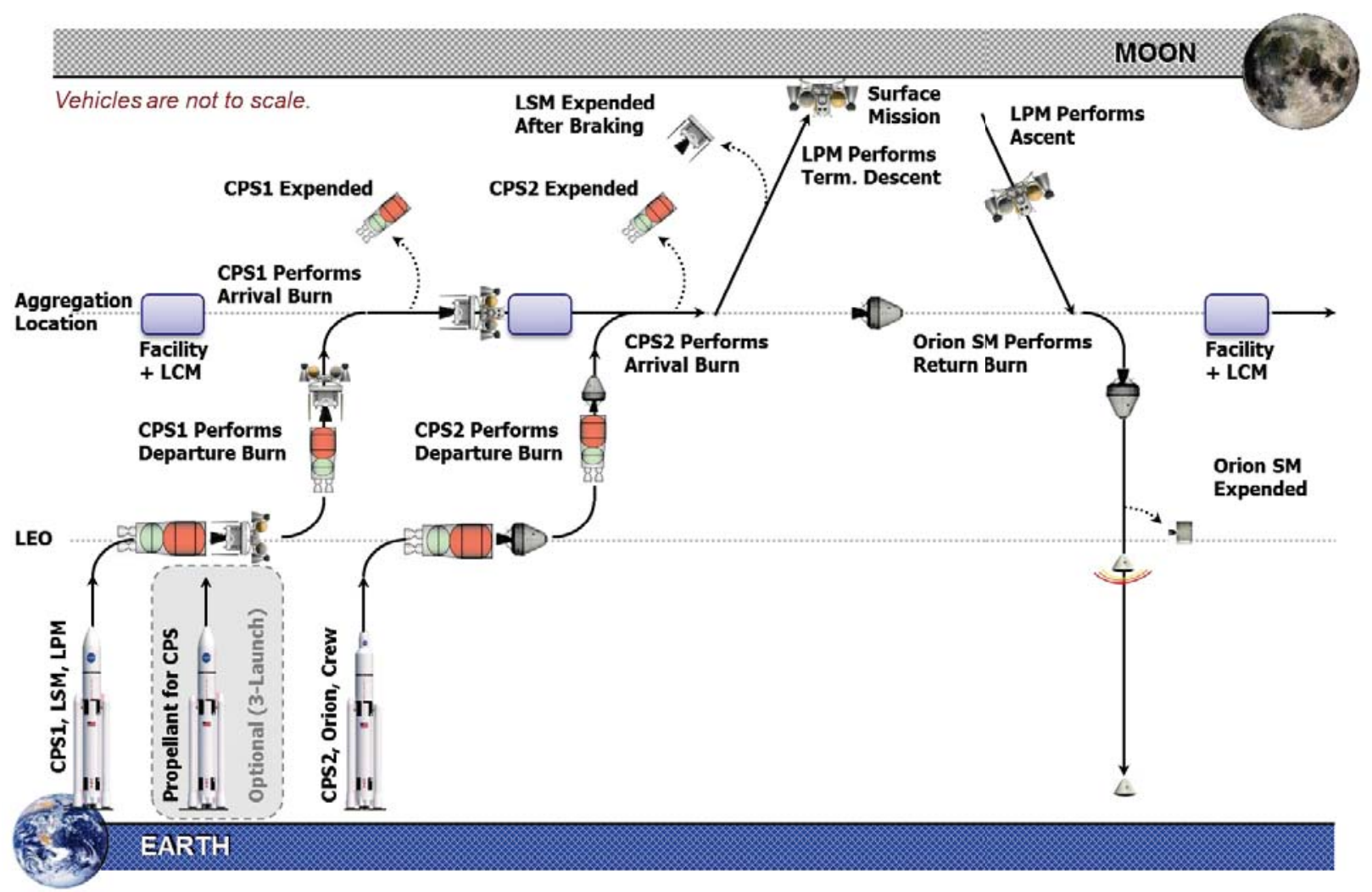

Figure 7: One-Stage Lunar Lander with Braking Stage Concept of Operations 


\section{Results and Discussion}

After exploring the options, analyzing over 800 architectures, presented above using a graph-based system architecture analysis framework [10], the required Propellant Mass Fraction (PMF) for the CPS is presented in the following section. The results are divided into the aggregation locations (LLO, HELO 1 and 2, and E-M L2), and the lunar lander configurations (two-stage, two-stage with OTV, and one-stage with braking stage) within each of those divisions.

\section{A. Low Lunar Orbit Aggregation}

The LLO aggregation places a large burden on the two CPSs to place the lunar lander and Orion deep into the lunar gravity well, but the lander performs a smaller $\Delta \mathrm{V}$ to get to the surface and back. The two-stage lunar lander with the OTV is not included in the LLO data set because the aggregation already occurs in LLO, and the OTV is unnecessary. Recall that the LCM is reusable and awaits the rest of the lunar lander and Orion at a facility in the aggregation orbit. Placing the facility in LLO could cause issues such as thermal cycling, orbit decay, and limited commercial access. Moving the facility farther from the Moon changes the eclipse cycle and reduces the requirements on the orbit insertion stage and station-keeping.

Figure 8 presents the required CPS PMF for the two-stage lunar lander configuration. The horizontal axis is the LPMD PMF, and the vertical axis is the minimum CPS PMF that fits onto the launch vehicle. The different curves represent different masses of the LCM and different LPMA PMF cases. Part (a) of the plot presents the two-launch, $105 \mathrm{t}$ launch vehicle scenario. The slope of these lines is fairly shallow, indicating that the CPS PMF is not very sensitive to LPMD PMF, whereas it is sensitive to the LPMA PMF as indicated by the large spacing between curves. Identifying the feasibility of a given CPS PMF is beyond the scope of this paper, but typical LOX/LH2 stages (Centaur, Delta Cryogenic Second Stage, Saturn S-IVB, etc.) have a PMF at or below approximately 0.9. These stages do not include the added mass for long storage duration and restarts. Therefore, while a given curve may not exceed the theoretical maximum of one, the scenario that the curve represents may still not be feasible. Throughout this paper, scenarios that have a minimum CPS PMF below one may be called "feasible," but the analysis to determine if creating a CPS to meet that minimum is not discussed.

Part (b) of Figure 8 presents the three launch, $105 \mathrm{t}$ launch vehicle scenario. Note that there are significantly more scenarios that are in the feasible space, including several with a $5 \mathrm{t} \mathrm{LCM}$ and LPMA PMF of 0.55. Also note again the increased sensitivity to LPMA PMF over LPMD PMF. Finally, part (c) of Figure 8 presents the threelaunch, $70 \mathrm{t}$ launch vehicle scenario. The reduction in launch vehicle mass only removes one curve (the one representing a $5 \mathrm{t}$ LCM and LPMA PMF of 0.55) when compared to the three-launch, $105 \mathrm{t}$ scenario. However, the minimum CPS PMF is increased by more than 0.1 in most cases.

It is noteworthy that the $53 \mathrm{t}$ missions to LEO are not included in any of the LLO aggregation results. That is due to the fact that one launch of a $53 \mathrm{t}$ launch vehicle cannot place the current Orion MPCV into LLO. The CPS (to get into LLO), CM, and SM cannot fit on a single $53 \mathrm{t}$ launch. Because multiple crew launches were not considered in this study, the $53 \mathrm{t}$ scenarios are infeasible, even though the lunar lander flights may be possible. 

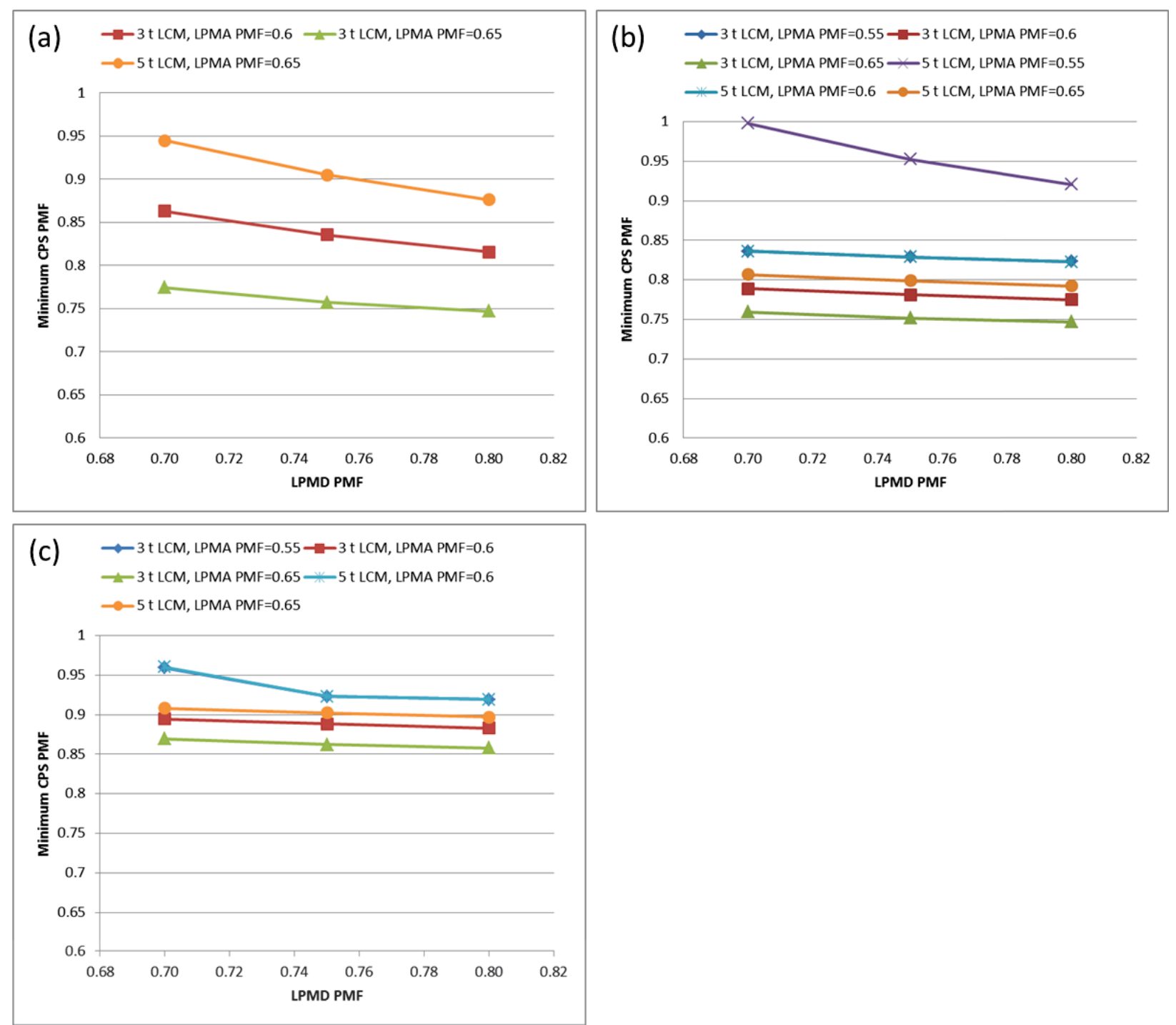

Figure 8: Two-Stage Lunar Lander Scenario for LLO Aggregation.

(a) 105 t, 2 Launch Scenario; (b) 105 t, 3 Launch Scenario; (c) 70 t, 3 Launch Scenario.

Figure 9 presents the required CPS PMF for the one-stage lunar lander with a braking stage configuration. The different curves represent different masses of the LCM and different LSM options (as-is Orion SM or LSS). Part (a) of the plot presents the two-launch, 105 t launch vehicle scenario. The slope of the LSS curves is shallower than the SM curves, indicating that the architectures that include the SM cannot overcome uncertainty or growth. Being able to vary the size of the LSS enables a more optimal $\triangle \mathrm{V}$ split for each LPM PMF.

Part (b) of Figure 9 presents the three launch, 105 t launch vehicle scenario. Note that all scenarios are included in the feasible space for both 2-launch and 3-launch scenarios. The curves are spaced closer together, indicating that the three-launch scenarios are more robust to growth in the LCM. Finally, part (c) of Figure 9 presents the threelaunch, $70 \mathrm{t}$ launch vehicle scenario. The reduction in launch vehicle mass keeps similar spacing to the three launch, 105 t scenario, but at increased CPS PMF. Again, for all three launch vehicle scenarios, using the Orion SM as the LSM is much more sensitive than using an LSS. 

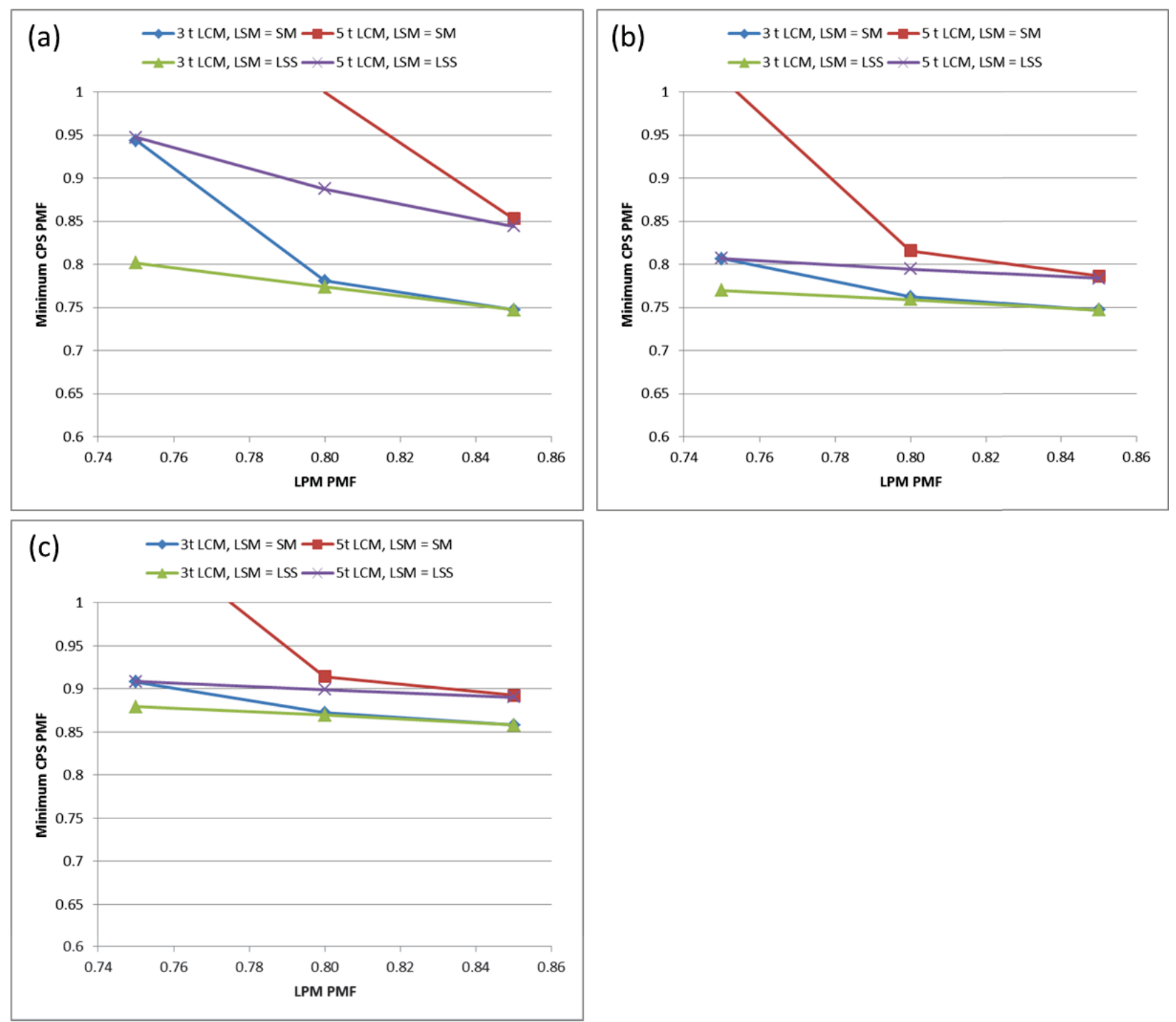

Figure 9: One-Stage Lunar Lander with Braking Stage for LLO Aggregation.

(a) 105 t, 2 Launch Scenario; (b) 105 t, 3 Launch Scenario; (c) 70 t, 3 Launch Scenario.

\section{B. High Elliptical Lunar Orbit Aggregation}

As the aggregation location moves further out of the lunar gravity well, more $\Delta \mathrm{V}$ burden is placed on the lunar lander, which is the highest gear ratio element. Therefore, the feasible space decreases the further the aggregation location is away from the Moon. This section presents the results from the analysis for both HELO aggregation locations, HELO 1 (100 x 1,000 km) and HELO 2 (100 x 10,000 km).

Figure 10 presents the required CPS PMF for the two-stage lunar lander configuration. The different curves represent different masses of the LCM and different values of LPMA PMF. Part (a) of the plot presents the threelaunch, $105 \mathrm{t}$ launch vehicle scenario. The scenarios that use two launches are all infeasible. The plot indicates again that the LPMA PMF is a primary driver for feasibility. The 0.55 LPMA PMF cases do not appear in the feasible range. Part (b) of Figure 10 presents the three launch, $70 \mathrm{t}$ launch vehicle scenario, and part (c) presents the three launch, $53 \mathrm{t}$ launch vehicle scenario. As the launch vehicle capability decreases, the minimum CPS PMF increases, and the scenarios with larger LCM masses become more infeasible, indicating how large of an effect the LCM mass has on the feasibility of the architecture. 

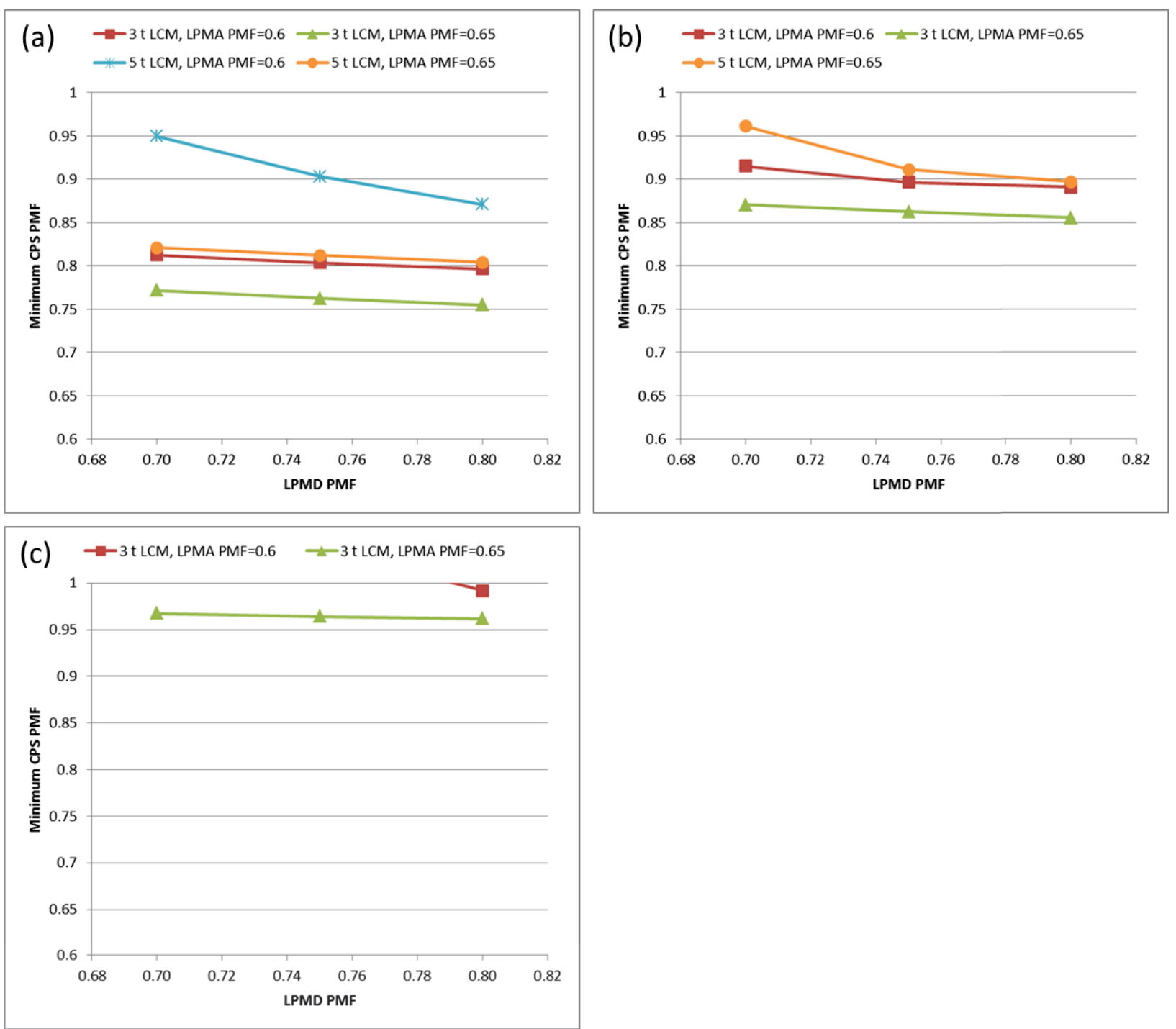

Figure 10: Two-Stage Lunar Lander Configuration for HELO 1 (100 x 1,000 km) Aggregation. (a) 105 t, 3 Launch Scenario; (b) 70 t, 3 Launch Scenario; (c) 53 t, 3 Launch Scenario. 
Figure 11 presents the required CPS PMF for the two-stage lunar lander configuration with an OTV. The different curves represent different masses of the LCM and different values of LPMA PMF. Part (a) of the plot presents the three-launch, $105 \mathrm{t}$ launch vehicle scenario, and part (b) presents the three launch, $70 \mathrm{t}$ and $53 \mathrm{t}$ launch vehicle scenarios. Adding an OTV to the two-stage lunar lander scenario increases the number of feasible design cases, enabling an LPMA with a PMF of 0.55.

Figure 12 presents the required CPS PMF for the one-stage lunar lander configuration with an braking stage. Part (a) presents the two- and three-launch scenarios of the $105 \mathrm{t}$ vehicle. The different curves represent different number of launches, masses of the LCM, and different LSM options (as-is Orion SM or LSS). The only feasible cases that use two launches also use a LSS for the braking stage. Using the LSS enables a more optimal $\Delta \mathrm{V}$ split between the LSM and LPM, which is based on the relative specific impulses (equal in this case) and PMF. Therefore as the LPM PMF decreases, the LSM performs more of the descent, up to a maximum of 90 percent. The analysis indicates that, for a less efficient LPM, the LSM should perform even more of the descent. The 90 percent maximum is used to protect for impact of elements on the surface and to provide time to troubleshoot any issues during separation. However, with more detailed analysis to determine the severity of these risks, this limit may be raised to perform more optimal staging. Part (b) presents the three launch, $70 \mathrm{t}$ scenario, and part (c) presents the three launch, $53 \mathrm{t}$ scenario. Reducing the launch vehicle capability increases the minimum CPS PMF, but all scenarios still exist in the feasible space.
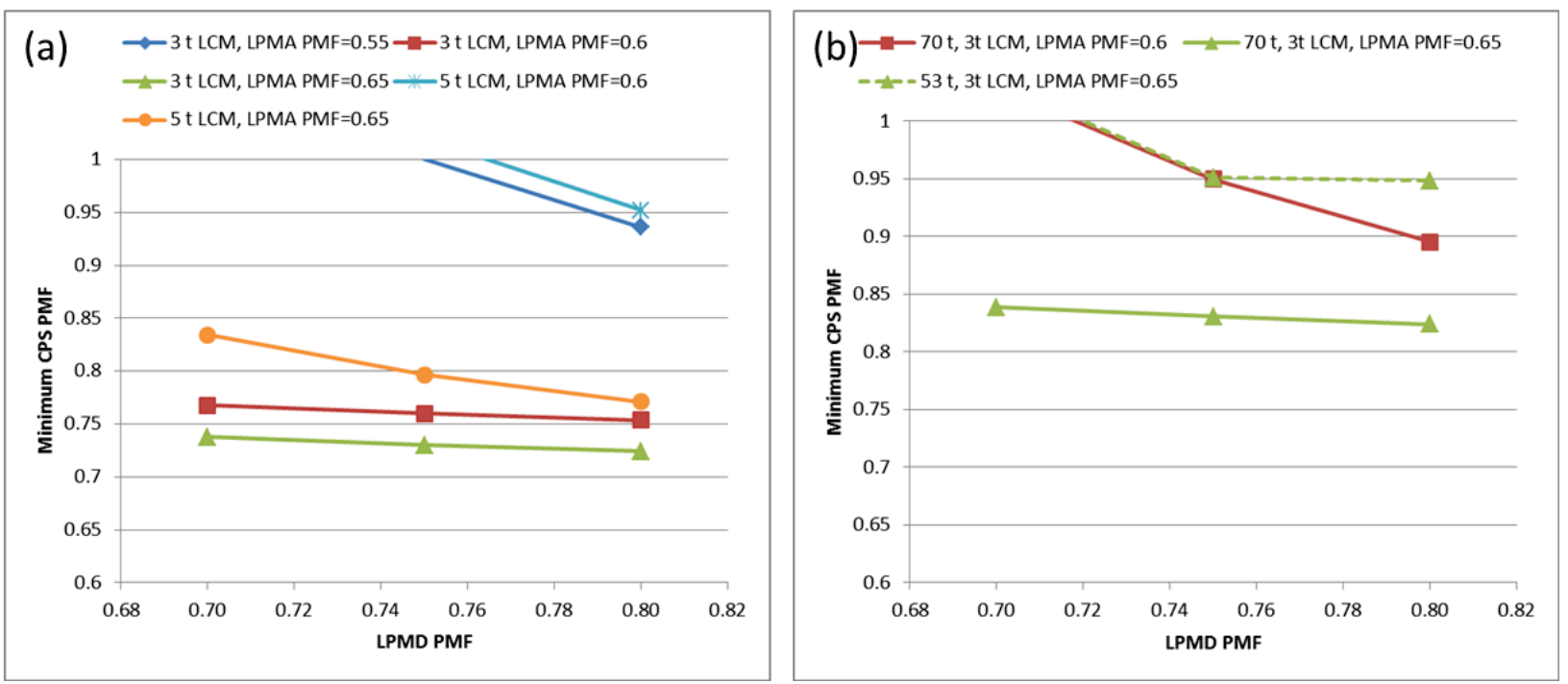

Figure 11: Two-Stage Lunar Lander with OTV Configuration for HELO 1 (100 x 1,000 km) Aggregation. (a) 105 t, 3 Launch Scenario; (b) 70 t and 53 t, 3 Launch Scenario. 

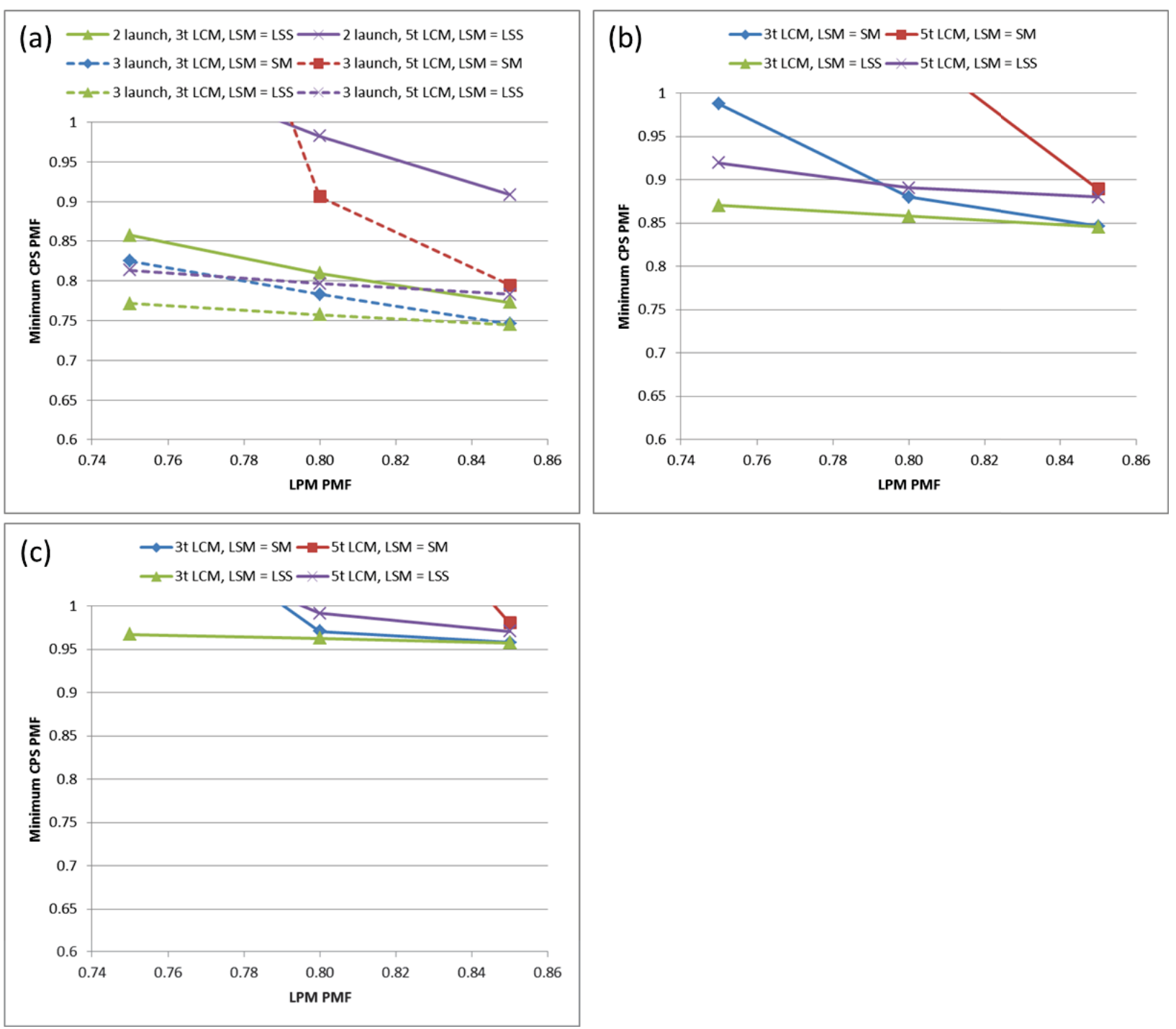

Figure 12: One-Stage Lunar Lander with Braking Stage Configuration for HELO 1 (100 x 1,000 km) Aggregation. (a) 105 t, 2 and 3 Launch Scenarios; (b) 70 t, 3 Launch Scenario; (c) 53 t, 3 Launch Scenario.

Figure 13 presents the two-launch, $105 \mathrm{t}$ launch vehicle scenario for the HELO 2 (100 x 10,000 km) aggregation location. Only the $3 \mathrm{t}$ LCM with a LPMA PMF of 0.65 scenario is feasible for each LPMD PMF. Again, the feasibility is not very sensitive to LPMD PMF. No feasible cases existed for the $70 \mathrm{t}$ or $53 \mathrm{t}$ launch vehicles. Figure 14 presents the two stage lunar lander configuration with an OTV. The $105 \mathrm{t}$ scenarios are feasible with a high LPMA PMF, and the $70 \mathrm{t}$ scenarios are feasible with a $3 \mathrm{t}$ LCM and a LPMA PMF of 0.65 . Finally, Figure 15 presents the one-stage lunar lander with braking stage. At this aggregation location, the LSS enables access to the lunar surface using all three launch vehicle types. 


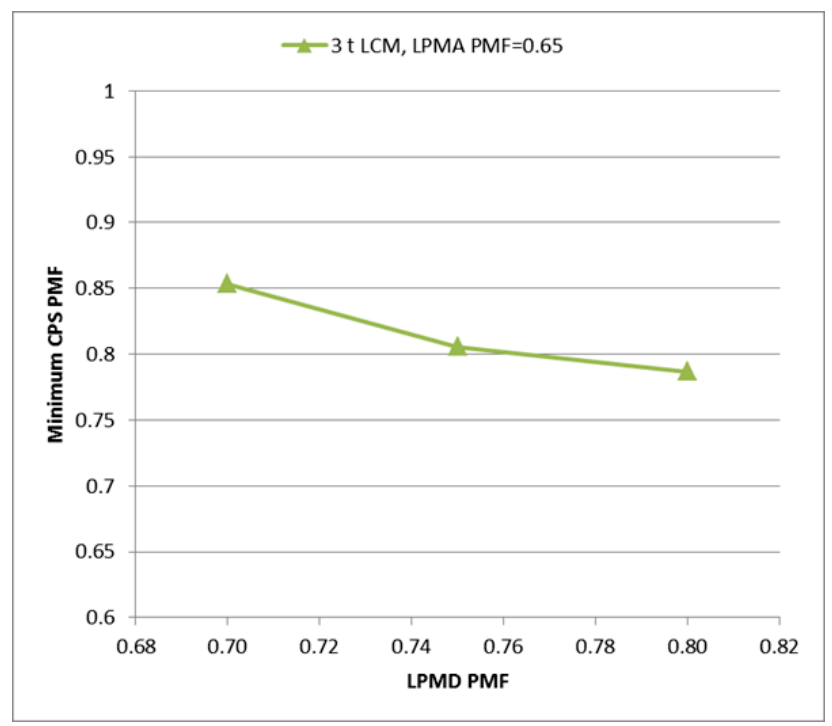

Figure 13: Two-Stage Lunar Lander Configuration for HELO 2 (100 x 10,000 km) Aggregation. 105 t, 3 Launch Scenario.
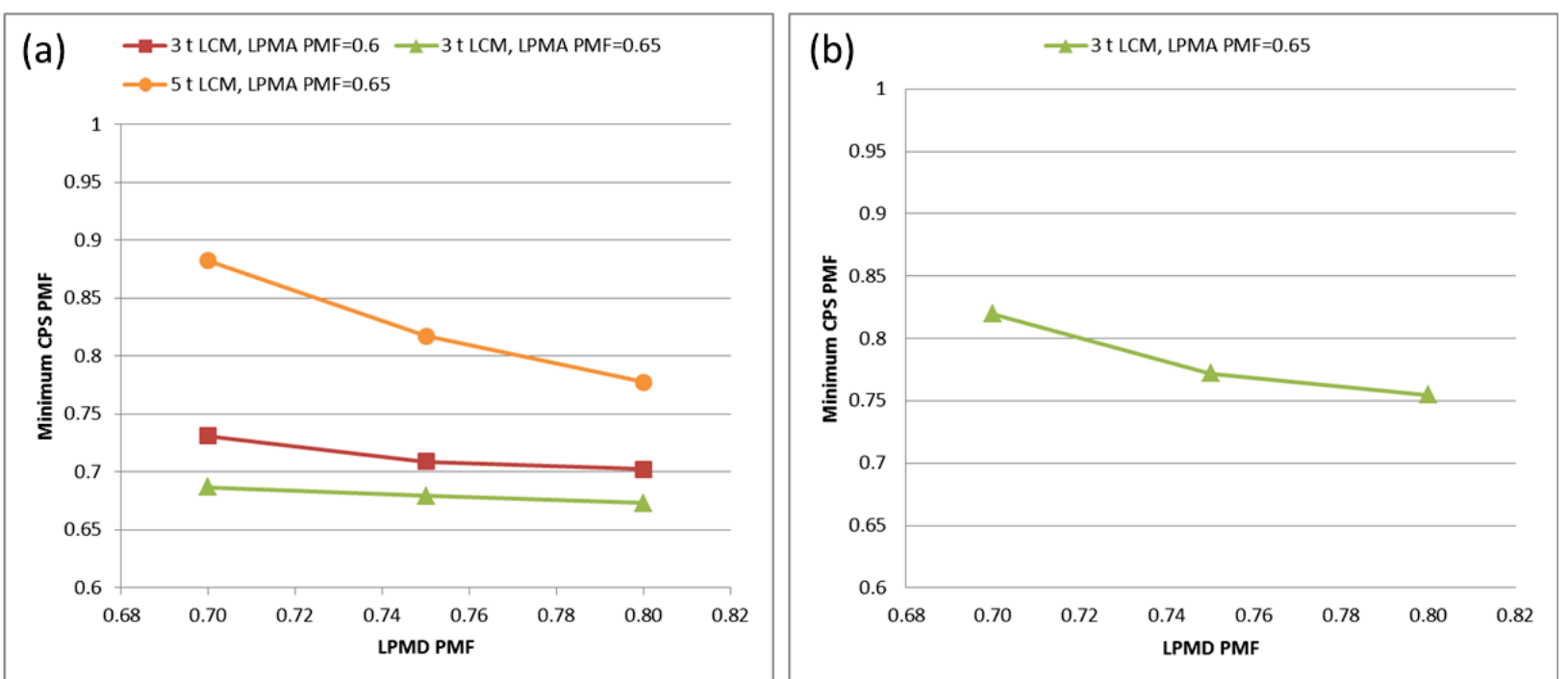

Figure 14: Two-Stage Lunar Lander with OTV Configuration for HELO 2 (100 x 10,000 km) Aggregation.

(a) 105 t, 3 Launch Scenario; (b) 70 t and 53 t, 3 Launch Scenarios. 

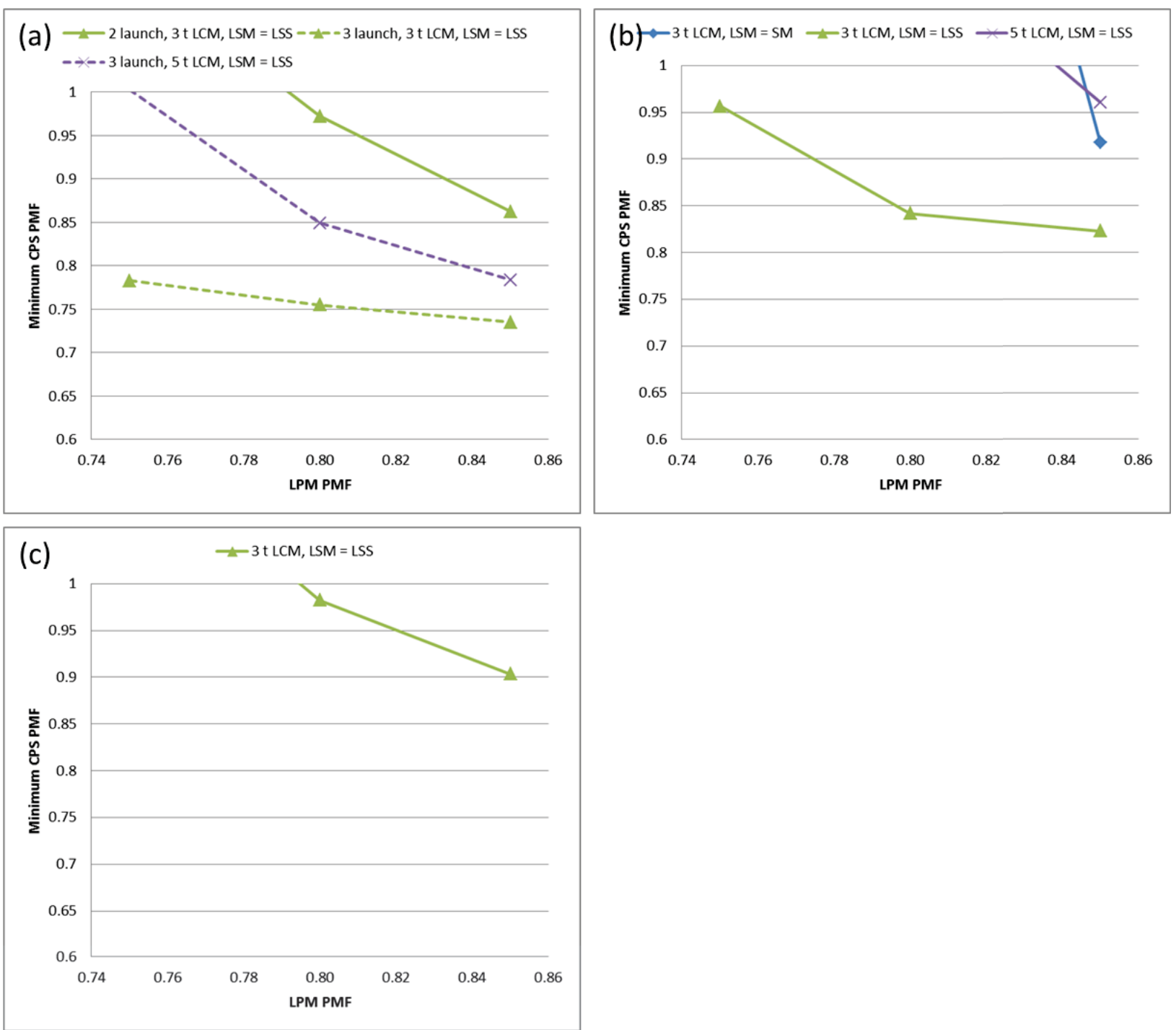

Figure 15: One-Stage Lunar Lander with Braking Stage Configuration for HELO 2 (100 x 10,000 km) Aggregation. (a) 105 t, 2 Launch and 3 Launch Scenarios; (b) 70 t, 3 Launch Scenario; (c) 53 t, 3 Launch Scenario.

\section{Earth-Moon L2 Aggregation}

The final aggregation location at Earth-Moon L2 (E-M L2) poses a great challenge to the lunar lander. It takes multiple days to travel from E-M L2 to the lunar surface, increasing the habitation demand to support the crew for longer duration. Also, the largest gear ratio element (the LPM or LPMA) must perform a much larger burn. For this reason, none of the two-stage lunar lander cases are feasible from E-M L2.

Figure 16 presents the results from the two-stage lunar lander with OTV configuration. Part (a) presents the three launch, $105 \mathrm{t}$ scenario, and part (b) presents the three launch, $70 \mathrm{t}$ scenario. Landers with a smaller LCM and high LPMA PMF are feasible with minimum CPS PMF values below 0.7. Figure 17 presents the one-stage lunar lander with braking stage configuration. The only feasible scenario is with the $3 \mathrm{t} \mathrm{LCM}$ and LSS, and the slope is very steep, indicating that this problem is very sensitive to LPM growth over the large required $\Delta \mathrm{V}$ to return to Earth-Moon L2. 

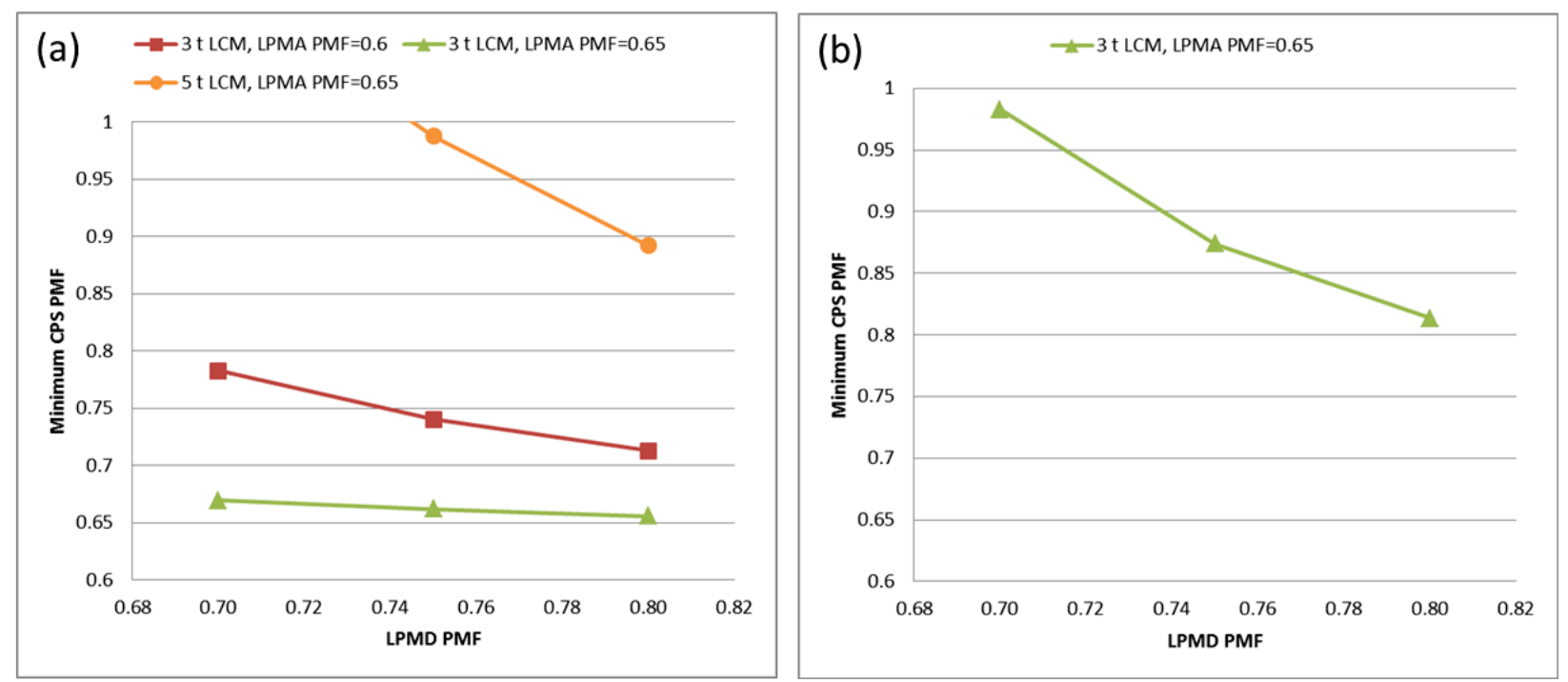

Figure 16: Two-Stage Lunar Lander with OTV Configuration for Earth-Moon L2 Aggregation Location.

(a) 105 t, 3 Launch Scenario; (b) 70 t, 3 Launch Scenario

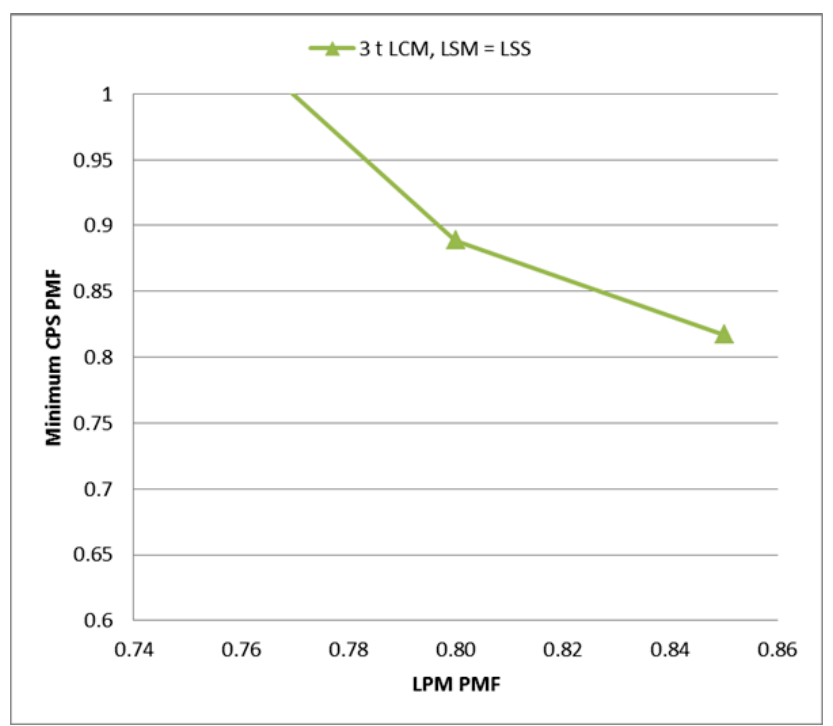

Figure 17: One-Stage Lunar Lander with Braking Stage Configuration for Earth-Moon L2 Aggregation Location.

\section{Conclusions}

This paper explored options within the lunar system architecture design space, identifying performance requirements placed on Cryogenic Propulsive Stage (CPS) based on existing and/or near-term capabilities. The analysis explored performance requirements for various system architecture options, including rendezvous and aggregation locations, launch manifests, and lunar lander configurations. The four aggregation locations assumed were Low Lunar Orbit (LLO), two High Elliptical Lunar Orbits, and Earth-Moon L2. The three launch vehicles considered in this study (105 t, $70 \mathrm{t}$, and $53 \mathrm{t}$ ) are consistent with the planned heavy lift vehicles for the near future, and depending on the scenario, both two and three launch scenarios were considered. Finally, three lunar lander configurations were considered: two-stage lander, two-stage lander with an Orbit Transfer Vehicle (OTV), and onestage lander with a braking stage.

In the two-stage lunar lander configurations, the ascent stage propellant mass fraction was a primary driver in creating a feasible architecture. In both the one- and two-stage lunar lander configurations, the Lander Crew Module mass was a primary driver. For the aggregation locations further from the lunar surface, a larger burden is 
placed on the lunar lander to access the surface, and these two metrics (which directly affect the lander mass) are more impactful.

Adding the OTV to a two-stage lunar lander increases the feasible design space as the aggregation location is moved farther out of the lunar gravity well. The one-stage lander with a braking stage configuration has a large feasible design space when the aggregation location is LLO. However, as the aggregation location moves further out, the Orion Service Module is insufficient, and the larger, more efficient stretched version of the Orion SM (Large Storable Stage) must be used. Also, the braking stage performs more of the descent as the Lander Propulsion Module has a less efficient propellant mass fraction.

\section{Acknowledgments}

The authors would like to acknowledge the encouragement and support by the excellent engineers in the Space Mission Analysis Branch and Vehicle Analysis Branch at NASA Langley Research Center, most notably D.R. Komar, Dan Mazanek, Matt Simon, and Min Qu.

\section{References}

1. Review of U.S. Human Spaceflight Plans Committee, Seeking a Human Spaceflight Program Worthy of a Great Nation, October 2009.

2. International Space Exploration Coordination Group, The Global Exploration Roadmap, NASA NP-2011-09-766-HQ, September 2011.

3. Singer, Jody, "Space Launch System (SLS) Program Overview, Advanced Development NASA Research

Announcement (NRA) Industry and Academia Day," NASA Marshall Space Flight Center, February 2012.

4. Falcon Heavy Overview Website, Space Exploration Technologies Corporation. URL: http://www.spacex.com/falcon_heavy.php. [retrieved 23 July 2013].

5. Orion Quick Facts. Lyndon B. Johnson Space Center, FS-2011-12-0.58-JSC.

6. "OME," Encyclopedia Astronautica, URL: http://www.astronautix.com/engines/ome.htm. [retrieved 23 July 2013 ].

7. Orloff, Richard W., Apollo by the Numbers: A Statistical Reference for the Manned Phase of Project Apollo, June 1996.

8. NASA's Exploration Systems Architecture Study Final Report. NASA TM-2005-214062. November 2005.

9. Mazanek, Daniel D., Goodliff, Kandyce E., and Cornelius, David M., "Descent Assisted Split Habitat Lunar Lander Concept.” IEEE Aerospace Conference, Paper 1384. March 2008.

10. Arney, Dale C. Rule-Based Graph Theory to Enable Exploration of the Space System Architecture Design Space. Ph.D. Dissertation, Georgia Institute of Technology. August 2012. 